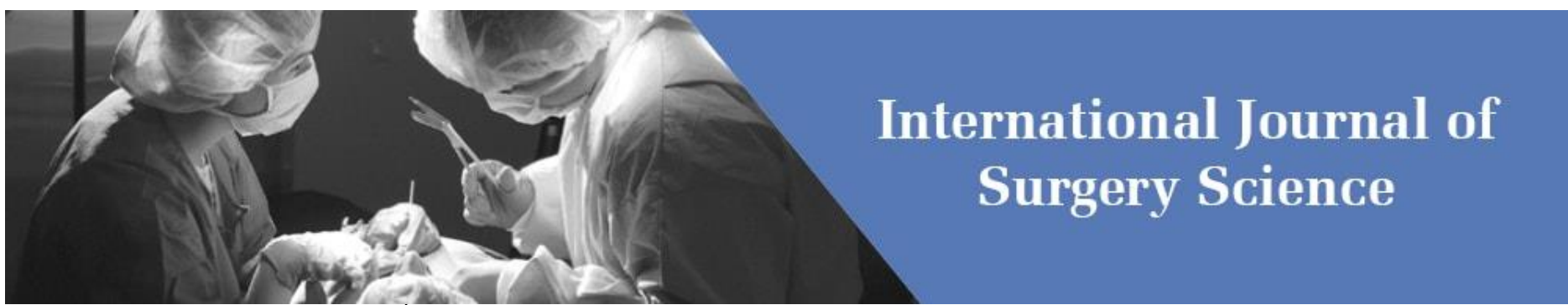

E-ISSN: 2616-3470

P-ISSN: 2616-3462

(C) Surgery Science

www.surgeryscience.com

$2019 ; 3(4): 226-229$

Received: 19-08-2019

Accepted: 23-09-2019

\section{Usharani Rathnam}

Assistant Professor, Department of Surgery, ESIC Medical College,

Post Graduate Institute of Medical Science and Research, Bangalore,

Karnataka, India

Santhosh Raju

Department of Surgery, ESIC

Medical College, Post Graduate

Institute of Medical Science and

Research, Bangalore, Karnataka,

India

Shivakumar Mallikarjun Algud Department of Surgery, ESIC

Medical College, Post Graduate

Institute of Medical Science and

Research, Bangalore, Karnataka, India

\section{Madhu Rajegowda}

Department of Surgery, ESIC

Medical College, Post Graduate

Institute of Medical Science and

Research, Bangalore, Karnataka, India

\section{Lakkanna Suggaiah}

Department of Surgery, ESIC Medical College, Post Graduate Institute of Medical Science and Research, Bangalore, Karnataka, India

\section{Analysis of stapled hemorrhoidopexy outcomes: A single-institution based study}

\section{Usharani Rathnam, Santhosh Raju, Shivakumar Mallikarjun Algud, Madhu Rajegowda and Lakkanna Suggaiah}

DOI: https://doi.org/10.33545/surgery.2019.v3.i4d.246

\section{Abstract}

Background: Although traditional surgery is the gold standard treatment for hemorrhoids, stapled hemorrhoidopexy $(\mathrm{SH})$ is an alternative surgical technique. However, this technique has concerns of recurrence. We conducted this study to assess the clinical outcomes and complications of SH in patients visiting our institution

Methods: A prospective study was conducted on 115 patients from 2010 to 2012 who underwent SH with PPH03 kit under spinal anesthesia. Clinical outcomes assessed included the operation time, hospital stay and rate of post-operative pain.

Results: SH had lower operative procedural time (30 minutes), post- operative pain and hospital stay (1.8 days) along with minimal procedural complications and were comparable to the previous reports.

Conclusions: Stapled hemorrhoidopexy is an effective alternative to traditional surgical technique in treating 3rd and 4th degree hemorrhoids, in terms of lesser operative procedural time, post-operative pain, use of analgesics and hospital stay along with reduced procedure related complication.

Keywords: Hemorrhoids; stapled hemorrhoidopexy; operative time; post-operative complication

\section{Introduction}

Hemorrhoids, commonly known as piles is a clinical condition affecting the anorectal region characterized by symptomatic enlargement and prolapsed anal cushion ${ }^{[1]}$. It is a common condition affecting the adults with a global incidence ranging between 50 to $80 \%$ and in India about $75 \%$ of the population are diagnosed with hemorrhoids ${ }^{[2]}$.

Conventional excisional hemorrhoidectomy has been the most effective technique for patients with hemorrhoids. However, the efficacy of the technique is masked by the complication of significant postoperative pain, thereby leading to the deferral of treatment ${ }^{[3]}$. To overcome this complication, Dr. Antonio Longo introduced stapled hemorrhoidopexy (SH) in 1998 and this procedure involves repositioning of the prolapsed hemorrhoidal tissue through a circular resection of the inner layers unlike the complete removal of the tissue followed in conventional methods ${ }^{[4]}$. Further the mechanical anopexy interrupts the vascular supply to the hemorrhoid cushions thereby reducing the hemorrhoid tissue ${ }^{[3]}$. $\mathrm{SH}$ is commonly indicated in circumferential grade II, hemorrhoidal prolapse, grade III and IV hemorrhoids ${ }^{[4]}$.

Evidences from clinical trials and meta-analysis studies have reported the safety and efficacy of SH in comparison to traditional excisional techniques ${ }^{[6,7]}$. The advantages of SH include shorter operative time, reduced hospital stay, lesser pain with earlier recovery. However, the higher symptomatic recurrence rate reported with $\mathrm{SH}$ has raised concerns over this procedure especially in cases of larger and prolapsed grade IV hemorrhoids ${ }^{[8]}$. Further, the eTHoS trial, a large, openlabel multicenter, randomized controlled trial demonstrated traditional excisional surgery as appropriate treatment of choice especially in a tailored management plan ${ }^{[9]}$. Based on negative effects reported with SH technique Giordano et al. suggested that the patients should choose the procedure either with higher risk of recurrence and additional operation or conventional hemorrhoidectomy associated with longer operation time and recovery time ${ }^{[10]}$.

In spite of the controversy regarding the use of $\mathrm{SH}$, it has been successfully used for the surgical management of hemorrhoids by many clinicians including our institution. However, surgical indications for SH may not be the same of conventional excisional techniques. The aim of the current study was to report the data regarding type of analgesia, post-operative morbidity,

\section{Usharani Rathnam}

Assistant Professor, Department of Surgery, ESIC Medical College,

Post Graduate Institute of Medica Science and Research, Bangalore,

Karnataka, India 
complication rate, the hospital stay, and the rate of recurrence observed with $\mathrm{SH}$ in our institutional experience.

\section{Materials and Methods}

This was a single-center prospective study conducted at ESIC MC PGIMSR, Bangalore. Data were collected prospectively for all consecutive patients with a diagnosis of hemorrhoids and were treated by SH in our institution between] 2010 and 2012.

Patients were included in the study if they had: i) symptomatic $2^{\text {nd }}, 3^{\text {rd }}$ or $4^{\text {th }}$ degree hemorrhoids and preferred undergoing $\mathrm{SH}$. Patients who reported acute hemorrhoidal episodes with thrombosis, prior hemorrhoidectomy and associated anal pathology were excluded from the study.

The medical history of all of the patients were recorded and the clinical examination included inspection, digital exploration and proctoscopy. Before the $\mathrm{SH}$, patients were advised to undergo routine blood and urine examinations.

The study protocol was approved by the institutional review board (IRB), while confirming to the standards of the Declaration of Helsinki and its subsequent revisions. All the included patients signed the informed consent to participate in the study.

\section{Treatment}

All the patients underwent SH under regional anesthesia as suggested by the anesthetist. The procedure was done in a lithotomy position. All the patients underwent $\mathrm{SH}$ using the procedure for prolapse and Haemorrhoids (PPH03) Proximate haemorrhoide stapler (Ethicon Endo-Surgery kit).

\section{Post-operative follow-up}

The follow up period ranged from 6 months to 32 months. All the patients were advised to have normal diet post operatively. They were also prescribed stool softener, mild analgesics and antibiotics for a period of 5 to 7 days.

Statistical analysis

\section{Statistical analysis}

All statistical analyses were performed using SPSS version 22.0 (IBM software suite; Armonk, NY). Data are expressed in its frequency and percentage as well as mean and standard deviation.

\section{Results}

\section{Baseline characteristics}

The study included a total of 115 patients with a mean age of 40 years (range: $21-70$ years). Majority of the patients were in the age group of 41 to 50 years and were predominantly males (M: $\mathrm{F}=70: 30$ ), figure 1 and 2 .

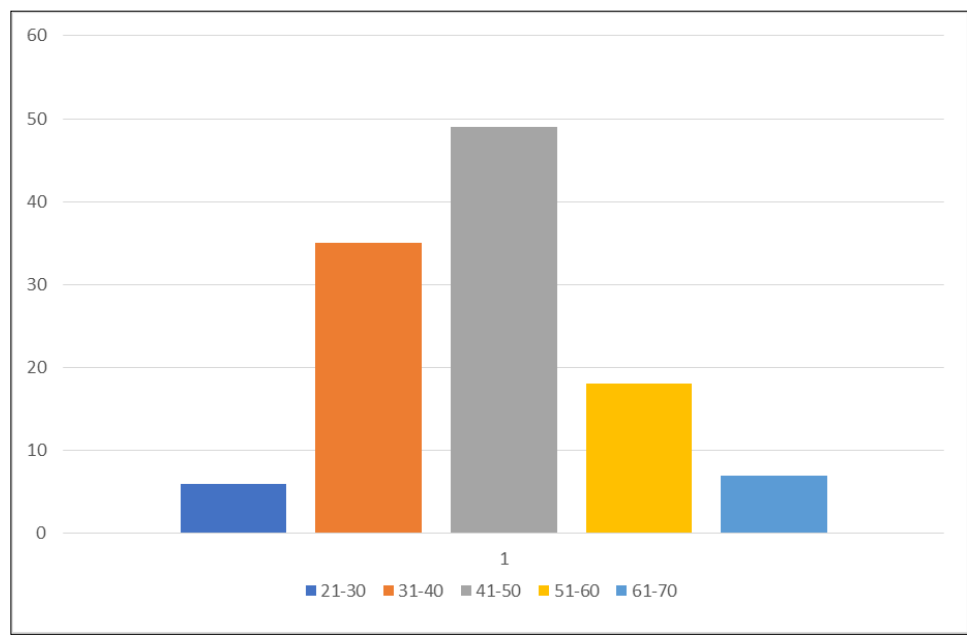

Fig 1: Incidence of hemmorrhoids according to age in the study population

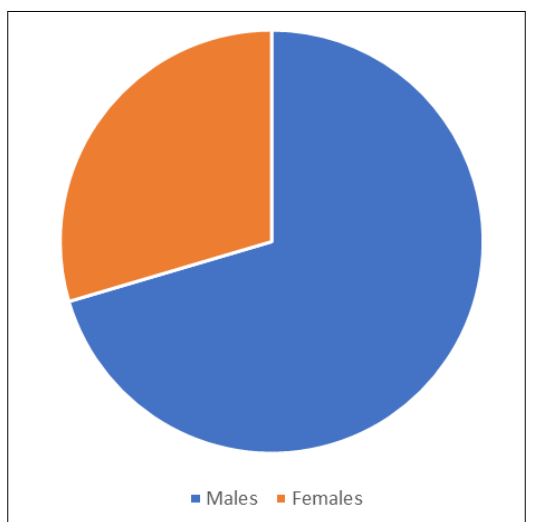

Fig 2: Incidence of hemmorrhoids according to gender in the study population

\section{Operation time}

The mean duration of surgery was 30 minutes, with a range of 25 to 45 minutes. About $81 \%$ of the patients had a surgery time of 30 to 35 minutes while the remaining patients either had $<30$ or $>35$ minutes of surgery time, figure 3 .

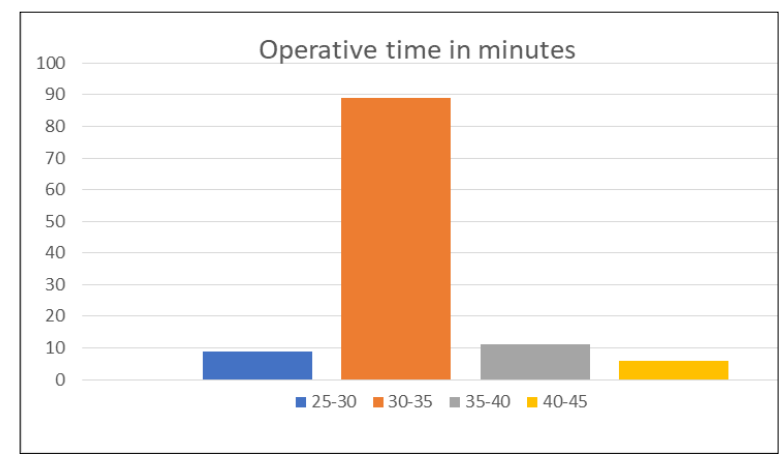

Fig 3: The different operative time within the Study Population

\section{Hospital stay}

The mean hospital stay in our study was 1.8 days. About $85 \%$ of the patients had $\leq 2$ days of hospital stay following $\mathrm{SH}$, while the remaining $15 \%$ of the patients had 2 to 5 days of hospital stay, figure 4 . 


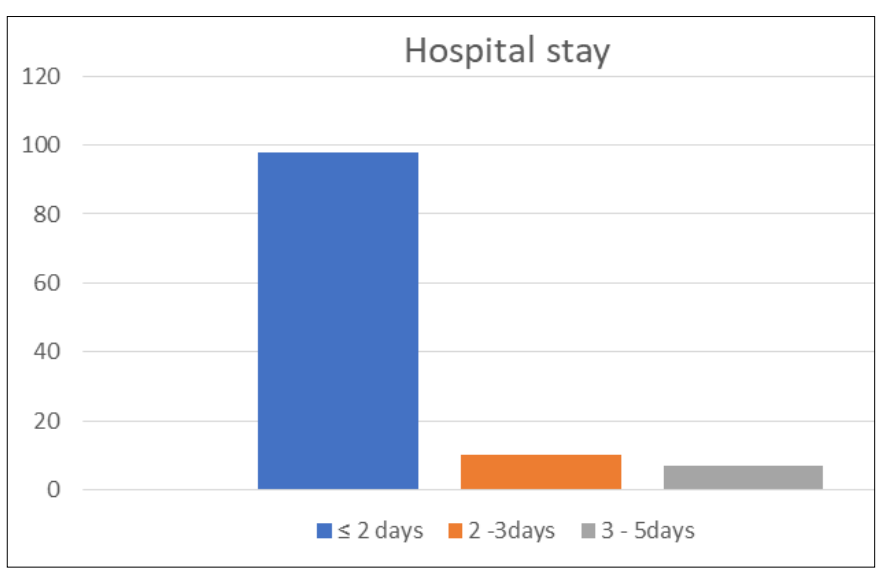

Fig 4: No of hospital stay in the Study Population

\section{Post-operative analgesia}

Pain was assessed using a visual analogue scale (VAS) in which 0 corresponds to "no pain" and 10 to "severe pain". In our study, most of the patients complained scheduled analgesia following SH and only $6.9 \%$ were reported severe pain and were treated with opiates.

\section{Post-operative complications}

In our study, about $9.5 \%$ of the patients reported post-operative bleeding, $11.3 \%$ had incontinence to flatus and $1.7 \%$ had faecal impaction. However, there were no cases of recurrences noted in our study. Further there were no other complications of rectal perforation and pelvic sepsis, rectal diverticulum and others as reported in other global studies.

\section{Discussion}

Hemorrhoids are one of the most common benign diseases and the management of this benign condition depends upon grade or degree of disease. Usually the grade I and II are treated by conservative measures while grade III and IV are managed surgically. Currently hemorrhoidal surgeries are performed by various intervention methods including Milligan-Morgan, Parks, and Ferguson and these procedures are still considered as gold standard treatment [11]. Stapled hemorrhoidopexy is an alternative to excisional surgery that is associated with clinical benefits including lesser surgical time and reduced time for recovery. This study was conducted to compare the results of $\mathrm{SH}$ performed in our institution with those reported in world literature.

The duration of surgery is less with $\mathrm{SH}$ in comparison to conventional methods. In our study the mean duration was about 30 minutes. This is similar to the duration reported by Panigrahi et al (28 minutes) but is higher than that reported by Vineet et al and $\mathrm{Ng} \mathrm{KH}$ et al where the surgery duration was 22 minutes and 15 minutes respectively [11, 12]. The varying difference in the duration of surgery may be due to the different grades of haemorrhoid patients included in different studies.

SH is usually performed as a day care procedure wherein the patients are discharged from hospital within a day. In our study, the mean hospital stay was 1.8 days. Our results were in line with that of Ganio et al, where they reported a mean hospital stay of 1 day ${ }^{[13]}$. Panigrahi et al reported a mean hospital stay of 2.08 days ${ }^{[5]}$. The slight differences noted in hospital stay with different studies is the reflection of differences in hospital discharge protocols and the way in which the length of hospital stay is determined in different studies.

The postoperative pain reported with $\mathrm{SH}$ is generally lesser compared to the traditional excisional biopsy. In our study only a limited number of patients had severe pain and had opiates had to be prescribed. Panigrahi et al had described a significantly lower pain score in SH patients compared to excisional method. Usually a purse string suture placed too close to the Dentate line or a low placed staple line can cause persistent postoperative pain following $\mathrm{SH}^{[14,15]}$.

In the current study, the common post-operative complication was incontinence to flatus followed by post-operative bleeding and faecal impaction. The rates or de novo incontinence to flatus reported in various prospective studies range between $3 \%$ to $19 \%[16,17]$. The use of anal dilator devices or stretching of the anal canal during insertion or firing of the stapler has been proposed as the cause of incontinence ${ }^{[18,20]}$. Further, the use of an Eisenhammer retractor for inserting purse string suture has been shown to reduce the incidence of incontinence ${ }^{[21,22]}$.

Another common complication of $\mathrm{SH}$ include bleeding, however this is lower when compared to the other methods of hemorrhoidectomy ${ }^{[23]}$. The rates of rectal bleeding after PPH for second-, third- and fourth-degree piles without thrombosis range between $1 \%$ and $11 \%{ }^{[16,17]}$ and in our study it was observed to be $9 \%$. Bleeding following stapled hemorrhoidopexy mostly occur immediately after surgery or later from the seventh day. Bleeding following SH usually occurs secondary to an arteriolar bleed along the staple line, either from defective techniques resulting in mucosal injury or due to the rejection of the staples ${ }^{[24]}$. The introduction of PPH03 has greatly reduced the incidence of early bleeding following $\mathrm{SH}$.

In our study a minor group of patients accounting $1.1 \%$ reported faecal impaction. However, some of the studies have reported an occurrence of about $1 \%$ to $6.6 \%{ }^{[25-28]}$.

Though previous studies have reported higher recurrences following $\mathrm{SH}$, in our study there was no cases of recurrence reported during the follow-up. Recurrence following $\mathrm{SH}$ has been reported up to $58.9 \%$ with a median recurrence rate of $6.9 \% .^{29}$ Grade IV hemorrhoidal disease is usually associated with recurrence. ${ }^{29}$ The meta-analysis study by Giordano et al reported recurrence rates following stapled hemorrhoidopexy in 4 th degree hemorrhoids up to $22 \%$, when compared to $3.6 \%$ in conventional hemorrhoidectomies ${ }^{[10]}$. Recurrence is believed to occur secondarily to the irreducibility of the prolapse preventing the lifting effect of the stapled hemorrhoidopexy ${ }^{[22,29]}$.

The limitation of our study is that it is a single arm study. We did not compare the $\mathrm{SH}$ procedure with the gold standard technique to get the definite difference between the two procedures.

\section{Conclusion}

In conclusion, stapled hemorrhoidopexy is a safe and efficacious surgical procedure in treating grade III and IV haemorrhoids. Most of the results obtained in our study were comparable with the currently available literature. However, the treating proctologist should be adequately and appropriately trained in this method of hemorrhoidectomy to achieve the best patient outcomes. Further studies with higher sample size reporting minimal recurrence are required to validate stapled hemorrhoidopexy as a gold standard treatment for hemorrhoids.

\section{References}

1. Lohsiriwat V. Hemorrhoids: from basic pathophysiology to clinical management. World J Gastroenterol. 2012; 18(17):2009-2017.

2. Ali SA, Shoeb MFR. Study of risk factors and clinical features of hemorrhoids. International Surgery Journal. 2017; 4(6):1936. 
3. Araujo SEA, Horcel L de A, Seid VE, Bertoncini AB, Klajner S. Long term results after stapled hemorrhoidopexy alone and complemented by excisional hemorrhoidectomy: A retrospective cohort study. Arq Bras Cir Dig. 2016; 29(3):159-163.

4. Ommer A, Hinrichs J, Möllenberg H, Marla B, Walz MK. Long-term Results After Stapled Hemorrhoidopexy: A Prospective Study With a 6-Year Follow-up: Diseases of the Colon \& Rectum. 2011; 54(5):601-608.

5. Panigrahi SK, Behera CR, Mishra S, Kanungo A. Stapled hemorrhoidopexy versus Milligan-Morgan haemorrhoidectomy: A paradigm shift in the management of $3^{\text {rd }}$ and $4^{\text {th }}$ degree hemorrhoids. International Surgery Journal. 2017; 5(1):209.

6. Bikhchandani J, Agarwal PN, Kant R, Malik VK. Randomized controlled trial to compare the early and midterm results of stapled versus open hemorrhoidectomy. The American Journal of Surgery. 2005; 189(1):56-60.

7. Boccasanta P, Capretti PG, Venturi M, et al. Randomised controlled trial between stapled circumferential mucosectomy and conventional circular hemorrhoidectomy in advanced hemorrhoids with external mucosal prolapse. Am J Surg. 2001; 182(1):64-68.

8. Cianci P, Altamura A, Tartaglia N, et al. Stapled hemorrhoidopexy: no more a new technique. Annals of Laparoscopic and Endoscopic Surgery. 2016; 1:25-25.

9. Watson AJM, Hudson J, Wood J, et al. Comparison of stapled haemorrhoidopexy with traditional excisional surgery for haemorrhoidal disease (eTHoS): a pragmatic, multicentre, randomised controlled trial. The Lancet. 2016; 388(10058):2375-2385.

10. Giordano P. Long-term Outcomes of Stapled Hemorrhoidopexy vs Conventional Hemorrhoidectomy: A Meta-analysis of Randomized Controlled Trials. Archives of Surgery. 2009; 144(3):266.

11. Singh S, Tayal A, Kaur V. Surgical Treatment of Haemorrhoids with Stapler Haemorrhoidopexy: Our Experince. JIMSA 2011; 24(2).

12. Ng K-H, Ho K-S, Ooi B-S, Tang C-L, Eu K-W. Experience of 3711 stapled haemorrhoidectomy operations. British Journal of Surgery. 2006; 93(2):226-230.

13. Ganio E, Altomare DF, Gabrielli F, Milito G, Canuti S. Prospective randomized multicentre trial comparing stapled with open haemorrhoidectomy: Stapled versus open haemorrhoidectomy. British Journal of Surgery. 2001; 88(5):669-674.

14. Seow-Choen F. Stapled haemorrhoidectomy: pain or gain: Leading article. British Journal of Surgery. 2001; 88(1):1-3.

15. Cheetham MJ, Mortensen NJ, Nystrom P-O, Kamm MA, Phillips RK. Persistent pain and faecal urgency after stapled haemorrhoidectomy. The Lancet. 2000; 356(9231):730-733.

16. Tjandra JJ, Chan MKY. Systematic Review on the Procedure for Prolapse and Hemorrhoids (Stapled Hemorrhoidopexy): Diseases of the Colon \& Rectum. 2007; 50(6):878-892.

17. Boccasanta P, Venturi M, Stuto A, et al. Stapled transanal rectal resection for outlet obstruction: a prospective, multicenter trial. Dis Colon Rectum. 2004; 47(8):12851296; discussion 1296-1297.

18. Uras C, Baca B, Boler DE. Circular stapled hemorrhoidopexy: experience of a single center with 445 cases. World J Surg. 2008; 32(8):1783-1788.

19. Mascagni D, Zeri KP, Di Matteo FM, Peparini N, Maturo A, Berni A. Stapled hemorrhoidectomy: surgical notes and results. Hepatogastroenterology. 2003; 50(54):1878-1882.

20. Ho YH, Tsang C, Tang CL, Nyam D, Eu KW, Seow-Choen F. Anal sphincter injuries from stapling instruments introduced transanally: randomized, controlled study with endoanal ultrasound and anorectal manometry. Dis Colon Rectum. 2000; 43(2):169-173.

21. Peng BC, Jayne DG, Ho Y-H. Randomized trial of rubber band ligation vs. stapled hemorrhoidectomy for prolapsed piles. Dis Colon Rectum. 2003; 46(3):291-297; discussion 296-297.

22. Finco C, Sarzo G, Savastano S, Degregori S, Merigliano S. Stapled haemorrhoidopexy in fourth degree haemorrhoidal prolapse: is it worthwhile? Colorectal Dis. 2006; 8(2):130134.

23. Chen J-S, You J-F. Current status of surgical treatment for hemorrhoids--systematic review and meta-analysis. Chang Gung Med J. 2010; 33(5):488-500.

24. Oughriss M, Yver R, Faucheron J-L. Complications of stapled hemorrhoidectomy: a French multicentric study. Gastroenterol Clin Biol. 2005; 29(4):429-433.

25. Ortiz H, Marzo J, Armendariz P. Randomized clinical trial of stapled haemorrhoidopexy versus conventional diathermy haemorrhoidectomy. Br J Surg. 2002; 89(11):1376-1381.

26. Ortiz H, Marzo J, Armendáriz P, De Miguel M. Stapled hemorrhoidopexy vs. diathermy excision for fourth-degree hemorrhoids: a randomized, clinical trial and review of the literature. Dis Colon Rectum. 2005; 48(4):809-815.

27. Goldstein SD, Meslin KP, Mazza T, et al. Stapled hemorrhoidopexy: outcome assessment. Am Surg. 2007; 73(7):733-736

28. Sobrado CW, Cotti GC de C, Coelho FF, Rocha JRM da. Initial experience with stapled hemorrhoidopexy for treatment of hemorrhoids. Arq Gastroenterol. 2006; 43(3):238-242.

29. Zacharakis E, Kanellos D, Pramateftakis MG, et al. Longterm results after stapled haemorrhoidopexy for fourthdegree haemorrhoids: a prospective study with median follow-up of 6 years. Tech Coloproctol. 2007; 11(2):144147; discussion 147-148. 